\title{
Physics of a Spinning Object Cyclic Inversion at an Orbital Flight
}

\author{
Ryspek Usubamatov ${ }^{1, *}$ and Marek Bergander ${ }^{2}$
}

\author{
${ }^{1}$ Kyrgyz State Technical University, Bishkek, Kyrgyzstan \\ ${ }^{2}$ AGH University of Science and Technology, Krakow, Poland
}

\begin{abstract}
The opening up of space flights is going on with physical discoveries. One of them was a spinning object cyclic inversion revealed on the MIR space station classified in 1985. Later, the NASA International Space Station openly showed the same effect. This physical effect was an object of stare studying by physicists and mathematicians. They developed only approximated and numerical models on the level of assumptions. The inversion of the spinning objects in the condition of free flight is the subject of gyroscope theory. The mass of the spinning object at the orbital flight generates the system of the interrelated inertial torques that results from the action of the inertial torques produced by the curvilinear motion of the object around the earth. This system of the torques acting on the spinning object at an orbital flight manifests its cyclic inversions, which is the gyroscopic effects. The theory of the gyroscopic effects describes the method of application of the system of the inertial torques, the physics of all gyroscopic effects that manifested by any rotating objects under any condition of their motions.
\end{abstract}

Keywords: Inertial toque, Gyroscope theory, Mathematical model, Inversion.

\section{INTRODUCTION.}

The orbital flights of men in space and their experimental investigations manifested the phenomenon of the rotational and cyclic inversions of the spinning objects. This phenomenon under the condition of the free flight of the spinning object shocked physicists because, at first sight, this effect contradicted the principles of classical mechanics [1-4]. The physicists and mathematicians of the entire world study this unusual effect [5-8]. They developed several approximated theories and numerical models, referred to the tennis racket theorem that did not bring the clarity and physical interpretation of the cyclic inversion of the spinning objects under the condition of the orbital flight. [9-11]. The detailed analysis of the spinning object cyclic inversions and its motions in space has shown a manifestation of the known gyroscopic effects at the process of its free flight. The physical principles of the gyroscope theory and classical mechanics [12-18] describe such motions of the spinning objects in space. The action of the inertial torque generated by the rotating mass of the spinning object under condition of the circular free flight and the weightless are manifested its gyroscopic effect of the cyclic inversion.

The number of publications dedicated to the physics of the cyclic inversion on orbital flights of the spinning objects is not so much. The cyclic inversions of the spinning object under conditions of the absence of the

*Address correspondence to this author at Kyrgyz State Technical University, Bishkek, Kyrgyzstan; Tel: +996 553722755;

E-mail: ryspek0701@yahoo.com; mark@mdienergy.com action of the external forces contradict the principles of physics. Only persistent researchers are studying this phenomenon. Analysis of their publications and methods for solutions show the application of the same mathematical tools and more intuitive assumptions as for the spinning top. Their principles for describing the spinning object inversion were only the angular momentum, kinetic energy, and the tennis racket theorem. The spinning object inversions on the orbital flight do not have the accepted physics and recognized mathematical model. The phenomenon of the cyclic inversion of the spinning object is described by the principles of the gyroscope theory and presented in this work.

\section{METHODOLOGY}

The mathematical model for motions of the spinning disc at the free flight is described by the principles of classical mechanics and the theory of gyroscopic effects. The rotating object around the fixed point is subjected by the action of the inertial torque. The gyroscope theory formulates the system of the inertial torques generated by the centrifugal, Coriolis forces, the change in the angular momentum of the spinning disc, and the ratio of its angular velocities around axes of rotation. These fundamental principles explain and describe the physics of acting forces and cyclic inversion of the spinning disc at the weightless.

For the analysis is considered the spinning disc of the horizontal location at the orbital flight. The axis of the disc disposed on the angle $\varphi$ to the line of its flight. The disc is spinning with the angular velocity $\omega$ in the counterclockwise direction around its axis. The flight of 
the disc is fulfilled with the constant tangential velocity $V$ around the earth at the distance $R$ from its center. The circular motion of the object generates the inertial torques that are acting on the object.

The textbooks of engineering mechanics presents the expression of the inertial torque acting on the object of the circular motion this is as follows: $T=\left(J_{0}+m r^{2}\right) \varepsilon$, where $J_{o}=J+m R^{2}$ is moment of inertia of the object defined by the parallel axis theorem; $J$ is the moment of inertia of the object around own center of mass; $m$ is the mas of the object; $R$ the radius of the object motion about the fixed point; $\varepsilon$ is the angular acceleration.

All freely rotating objects always turn about their center mass with the angular velocity of their rotation about the fixed point. The confirmation of this statement is the circular motion of the moon that always shows its one side toward to the earth.

The kinetic energy of the circular orbital flight of the disc produces the inertial torques that turn the vector of the tangential velocity $V$ of the disc toward the earth. The expression of the inertial torque that are external is as follows:

$T=J_{o} \omega_{o}^{2}=\left(\frac{m r^{2}}{2}+m R^{2}\right)\left(\frac{V}{R}\right)^{2}=m\left(\frac{r^{2}}{4}+R^{2}\right)\left(\frac{V}{R}\right)^{2}$

where $r$ is the radius of the disc; $R$ is the orbital radius of the disc flight; $\omega_{o}=V I R$ is the orbital angular velocity of the disc flight about the earth's center; other parameter are as specified above.

The external torque $T$ produces the system of the interrelated inertial torques generated by the rotating mass of the spinning object $[12,13]$ are presented in Table 1.
Table 1: Equations of the Inertial Torques Acting on the Rotating Disc

\begin{tabular}{|c|c|}
\hline $\begin{array}{c}\text { Type of the Torque Generated } \\
\text { by }\end{array}$ & Equation, $\mathbf{k g} \cdot \mathbf{m}^{2} / \mathbf{s}^{2}$ \\
\hline \hline Centrifugal forces, (axis ox) & $T_{c t}=\frac{4}{9} \pi^{2} J \omega \omega_{x}$ \\
\hline Centrifugal forces, (axis oy) & $T_{c r}=\frac{8}{9} J \omega \omega_{i}$ \\
\hline Coriolis forces & $T_{a m}=J \omega \omega_{i}$ \\
\hline Change in angular momentum & $T_{r}=\left(\frac{4 \pi^{2}+8}{9}\right) J \omega \omega_{x}$ \\
\hline $\begin{array}{c}\text { Resistance torque } T_{r}=T_{c t}+T_{c r} \\
\text { Precession torque } T_{p}=T_{c t}+T_{a m}\end{array}$ & $T_{p}=\left(\frac{4 \pi^{2}+9}{9}\right) J \omega \omega_{x}$ \\
\hline $\begin{array}{l}\text { The dependency of angular velocities of the disc rotation about } \\
\text { axes oy and ox, degrees/s }\end{array}$ \\
\hline \multicolumn{2}{|c|}{$\omega_{y}=\left(8 \pi^{2}+17\right) \omega_{x}$} \\
\hline
\end{tabular}

Where $\omega_{i}$ is the angular velocity of the rotation of the disc about axis $i$; other parameter are as specified above.

Two system of external and inertial torques (Eq. 1) and Table 1) are used for the mathematical modeling of the spinning disc motions and the cyclic inversions at the orbital flight. The combined action of the inertial torques and motions around three axes for the spinning disc before the first inversion are represented in Figure 1. The action of the external torque $T$ changes direction of the vector of the angular momentum $H$ of the spinning disc on the plane, yoz that presented by $H_{x}$, and on the plane, $x O z$ by $H_{y}$. The angular momentums on these planes produce their own system of inertial torques of the spinning (Figure 1).

The inertial torques (Table 1) of the spinning disc about axes are presented by the following components (Figure 1):

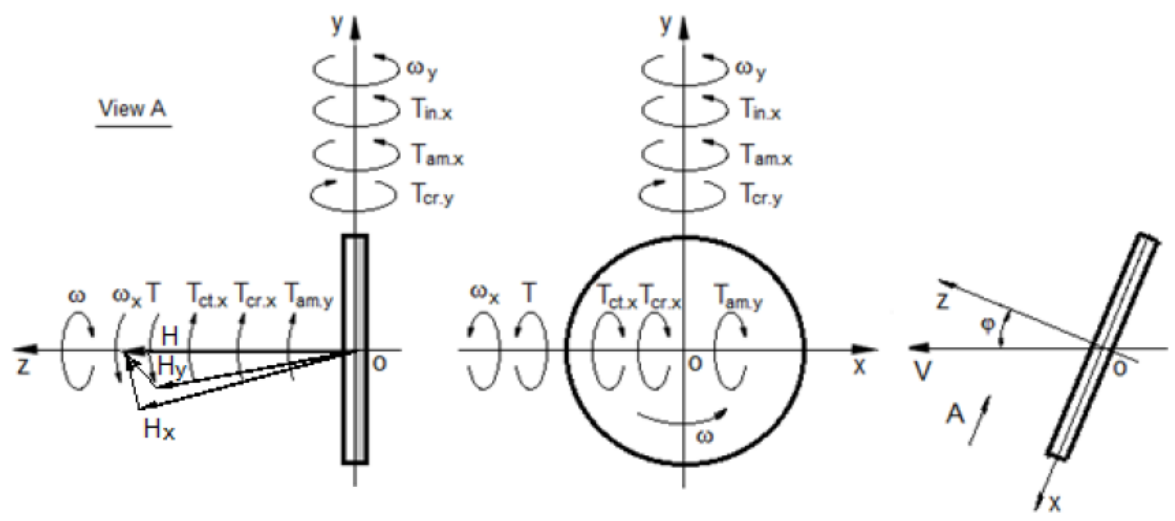

Figure 1: Schematic of the torques acting on the spinning disc and its motions at the orbital flight. 
-The external torque $T$ (Eq. (1)) is the result of the orbital circular flight that produces the resistance torque $T_{x}=-T_{c t x}-T_{c r x}-T_{i n . y}-T_{a m y}$

-The resulting torque around axis ox produces the resulting precession torque about axis oy $T_{y}=T_{c t x}+T_{a m x}-T_{c t . y}-T_{c r y}$

This the action of external and inertial torques on the spinning disc enables describing and explain the physics of inversion at the orbital flight for two conditions, i.e., before the first inversion and after the second one. The method for formulating of the mathematical model for the motions of the spinning disc is presented in several publications $[12,13]$ that used for the modeling of the cyclic inversions of the spinning disc. The mathematical model for the first inversion is expressed by the following Euler's differential equations $[12,13]$ :

$J_{x} \frac{d \omega_{x}}{d t}=T \cos \varphi-T_{c t x}-T_{c r x}-T_{c t . y}-T_{a m y}$

$\pm J_{y} \frac{d \omega_{y}}{d t}=T_{c t x}+T_{a m x}-T_{c t . y}-T_{c r y}$

$\pm \omega_{y}=\left(8 \pi^{2}+17\right) \omega_{x}$

where $\omega_{x}$ and $\omega_{y}$ is the angular velocity of the spinning disc about axes ox and oy, respectively; $t$ is the time; $\varphi$ is the angle of the inclination of the spinning disc axle to the line of its orbital flight (Figure 1) on the horizontal plane xoy; $J_{y}$ and $J_{x}$ are the mass moments of inertia of the disc about axes oy and ox, respectively; other components are as specified in Table 1.

Substituting expressions of Table 1 and the torque $T$ (Eq. 1) into (Eq. 2) yield the following equation:
$J_{x} \frac{d \omega_{x}}{d t}=m\left(\frac{r^{2}}{4}+R^{2}\right)\left(\frac{V}{R}\right)^{2} \cos \varphi-\frac{4}{9} \pi^{2} J \omega \omega_{x}-$

$\frac{8}{9} J \omega \omega_{x}-\left[8 \pi^{2}+17\right] J \omega \omega_{x}$

Equations (5) and (4) enable computing the angular velocity of the spinning disc about axis ox and oy respectively.

Simplification and transformation of Eq. (5) yields the following:

$$
\begin{aligned}
& J_{x} \frac{d \omega_{x}}{d t}=m\left(\frac{r^{2}}{4}+R^{2}\right)\left(\frac{V}{R}\right)^{2} \cos \varphi- \\
& \left(\frac{76 \pi^{2}+161}{9}\right) J \omega \omega_{x}
\end{aligned}
$$

where all other components are as specified above.

The value of the angular velocities $\omega_{x}$ and $\omega_{y}$ of the spinning disc depends on the angle $\varphi$ of the disc axis inclination to the line of orbital flight. If the disc axis is perpendicular to the line of the flight, the external toque $T$ does not generate the inertial torques of precession and the spinning disc does not manifest the inversion. The vertical or intermediate angular location of the disc axis to the line of its flight produces the external torque $T$ and the inertial torques of precession and the inversion of the spinning disc.

The spinning disc turns in a counter-clockwise direction intensively about axis oy (Eq. 5). The spinning disc turns on $90^{\circ}$, the precision torque is nullified but inertial forces turns the disc until $180^{\circ}$. The disc stops because the external torque $T$ generates the precession torque acting opposite direction (Figure 2). The spinning disc turns in a clockwise direction about axis oy.
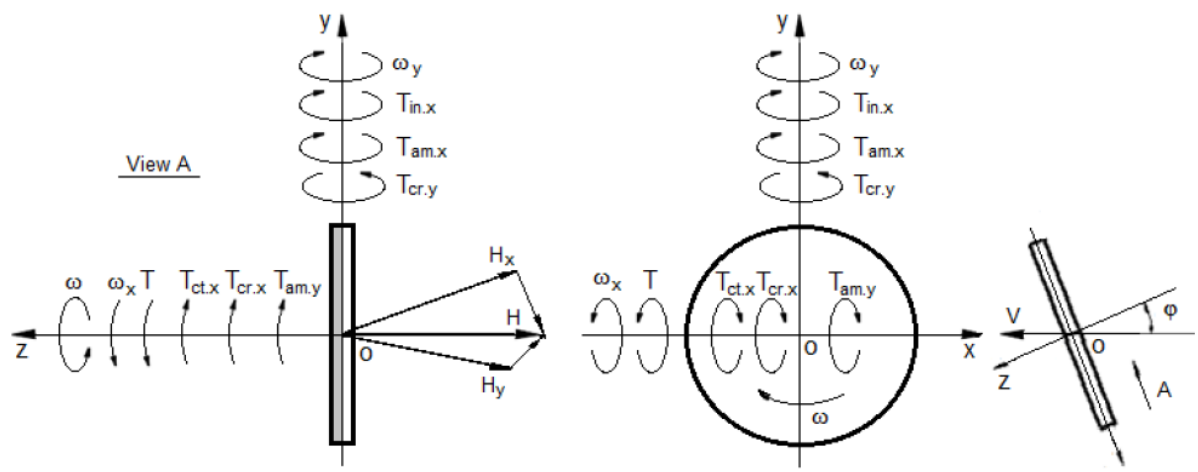

Figure 2: Schematic of the torques acting on the spinning disc and its motions at the orbital flight before the second inversion. 
The equation of the turn for the second inversion of the spinning disc about axis ox is presented by the following:

$$
\begin{aligned}
& -J_{x} \frac{d \omega_{x}}{d t}=m\left(\frac{r^{2}}{4}+R^{2}\right)\left(\frac{V}{R}\right)^{2} \cos \varphi- \\
& {\left[\frac{76 \pi^{2}+161}{9}\right] J \omega \omega_{x}}
\end{aligned}
$$

where the sign (-) of the left side equation means the clockwise direction of the turn.

The spinning disc turns back on $180^{\circ}$ and the following action of the torques and motion are repeated. The value of the external torque $T$ and the frequency of inversions will decrease with an increase in the angle of the angle $\varphi$.

\section{CASE STUDY AND WORKING EXAMPLE}

The orbital flight of the spinning disc is caring out with a velocity of $7844 \mathrm{~m} / \mathrm{s}$ and above the earth's surface on $1,0 \times 10^{5} \mathrm{~m}$. The radius of the orbital flight is $R=6,3781 \times 10^{6}+1,0 \times 10^{5}=6,4781 \times 10^{6} \mathrm{~m}$. The axis of the spinning disc coincided with the direction of the orbital flight. The mass of the disc is $0.1 \mathrm{~kg}$, radius of $0,05 \mathrm{~m}$, the spinning is $500 \mathrm{rpm}$. The moment of inertia of the disc is $J=m r^{2} / 2=0,1 \times 0,05^{2} / 2=1,25 \times 10^{-4} \mathrm{~kg} \cdot \mathrm{m}^{2}$; the moment of inertia about the diametric line is $J_{x}=$ $\mathrm{mr}^{2} / 4==0,1 \times 0,05^{2} / 4=6,25 \times 10^{-5} \mathrm{~kg} \cdot \mathrm{m}^{2}$; and the moment of inertia for orbital flight is $J_{x o}=m r^{2} / 4+m R^{2}=$ $0,1 \times 0,05^{2} / 4+0,1 \times\left(6,4781 \times 10^{6}\right)^{2}=4,196577961 \times 10^{12}$ $\mathrm{kg} \cdot \mathrm{m}^{2}$. This flight of the spinning disc is considered for the two conditions that are before the first and the second inversion.

Substituting obtained data into Eq. (6) and (5) yield the following equations of the spinning disc motion around axis ox and oy.

The angular velocity of the spinning disc about axis ox before the first inversion is

$$
\begin{aligned}
& 6,25 \times 10^{-5} \frac{d \omega_{x}}{d t}=4,196577961 \times 10^{12} \times \\
& \left(\frac{7844}{6,4781 \times 10^{6}}\right)^{2} \times \cos 0^{o}- \\
& {\left[\frac{76 \pi^{2}+161}{9}\right] \times 1,25 \times 10^{-4} \times 500 \times \frac{2 \pi}{60} \omega_{x}}
\end{aligned}
$$

Simplification of Eq. (8) yields the following:

$$
6,25 \times 10^{-5} \quad \frac{d \omega_{x}}{d t}=6152833,6-0,662563 \omega_{x}
$$

Separating variables of Eq. (9) and presenting by the integral form yield the following:

$$
\int_{0}^{\omega_{x}} \frac{d \omega_{x}}{9286408,745-\omega_{x}}=-10601,012 \int_{0}^{t} d t
$$

The left integral of Eq. (10) is tabulated and represented the integral $\int \frac{d x}{a-x}=-\ln x+C$. The right integral is simple. Solving of integrals yields the following equation:

$$
\left.\ln \left(9286408,745-\omega_{x}\right)\right|_{0} ^{\omega_{x}}=-10601,\left.012 t\right|_{0} ^{t}
$$

that gave rise to the following

$$
1-\frac{\omega_{x}}{9286408,745}=e^{-10601,012 t}
$$

The right component of Eq. (11) has a small value of high order that can be neglected.

Then Eq. (11) yields the following result:

$\omega_{x}=9286408,745 \mathrm{rad} / \mathrm{s}$

The angular velocity around axis oy (Eq. 5) is:

$$
\begin{aligned}
& \omega_{y}=\left[8 \pi^{2}+17\right] \omega_{x}= \\
& {\left[8 \pi^{2}+17\right] \times 9286408,745=} \\
& 8,910 \times 10^{9} \mathrm{rad} / \mathrm{s}
\end{aligned}
$$

The angular velocities of the spinning disc for the second inversion are the same as for the first one. The process of the spinning disc motion with an inversion at the orbital flight is repeated.

The angular velocity of the spinning disc about axes $o x$ and oy at the orbital flight has high values. These motions are executed by the start-stop regime. Cosine law changes the value of the inertial torque acting on the spinning disc about axis oy. The disc turns on $90^{\circ}$ nullifies the value of the inertial torque and its following turn increases the value that acting in opposite direction (Figure 2). These motions of the spinning disc are fulfilled with the high angular velocities at a short time and present the damped harmonic oscillations about axes ox and oy.

\section{RESULTS AND DISCUSSION}

The mathematical model for the motions and cyclic inversion of the spinning disc at the orbital flight derived on the principles of the theory of gyroscopic effects for rotating objects $[8,9]$. The inversion process of the 
spinning disc is the result of the action of the system of the interrelated inertial torques generated by its rotating mass. The known principles of the classical mechanics show the circular motion of the object about the fixed point produces the inertial torques that turns the object about its center mass. The flight of the object around the earth is carrying out by circular motion. These combined inertial torques act on the spinning disc at the condition of the orbital flight manifest its cyclic inversions and damped harmonic oscillations. If the axle of the spinning objects is parallel to the axis of action of the external torques, they do not manifest the gyroscopic effects. The intensification of the gyroscopic effects of the spinning object at the orbital flight depends on the angle of the inclination of the object axel to the direction of the flight, t.e., to the axis of the action of the external torques. The derived mathematical model describes the physics of the spinning disc cyclic inversions at the orbital flight by the principles of classical mechanics.

\section{CONCLUSION}

The theory of gyroscopic effects for the spinning objects can solve all problems based on the action of the system of the interrelated inertial torques that are generated by the rotating mass. The spinning object at the orbital flight demonstrated its cyclic inversions that is a gyroscopic effect at the weightlessness and the result from the action of the inertial torques generated by its curvilinear motion and the inertial torques of the gyroscope theory. The inertial torques acting on the object express the kinetic energy of the object at the circular motion. The solution of the cyclic inversions of the spinning object at the orbital flight is based on the principles of classical mechanics and presents a good example of the educational process.

\section{REFERENCES}

[1] Petrov AG \& Volodin SE. Janibekov's effect and the laws of mechanics, Doklady Physics, Mechanics, Moscow, 2013; 58: 349-353. https://doi.org/10.1134/S1028335813080041

[2] Murakami $\mathrm{H}$, Rios $\mathrm{O}$, Impelluso T. A Theoretical and Numerical Study of the Dzhanibekov and Tennis Racket Phenomena, Journal of Applied Mechanics 2016. https://doi.org/10.1115/IMECE2015-52374
[3] Trivailo PM and Kojima H. Discovering Method of Control of the "Dzhanibekov's Effect" and Proposing it's Applications for the Possible Future Space Missions, Transactions of the Japan society for aeronautical and space sciences, Aerospace Technology Japan, 2019; 17 (1): 72-81, https://doi.org/10.2322/tastj.17.72

[4] Trivailo PM and Kojima $\mathrm{H}$, Enhancement of the attitude dynamics capabilities of the spinning spacecraft using inertial morphing, The Aeronautical Journal, 2019; 124(1276); June 2020; pp. 838-871

https://doi.org/10.1017/aer.2019.145

[5] Zhuravlev VF and Rozenblat GM. Estimates of Solutions During Motion of the Euler -Poinsot Top and Explanation of the Experiment with Dzhanibekov's Nut, Rus. J. Nonlin. Dyn 2020; 16(3): 517-525.

https://doi.org/10.20537/nd200308

[6] Tello RZ. El Effect Dzhanibekov, Fisica, ITAM, 2020; No. 51, (Spain).

[7] Mardesic P, Van Damme L, Gutierrez Guillen GJ, Sugny D. Geometric Origin of the Tennis Racket Effect, Phys. Rev. Lett. 2020; 125: 064301,

https://doi.org/10.1103/PhysRevLett.125.064301

[8] Ashbaugh MS, Chicone CC, and Cushman RH, The Twisting Tennis Racket, (1991) Journal of Dynamics and Differential Equations 3(1), pp. 67-85. https://doi.org/10.1007/BF01049489

[9] R. van Zon and Schofield J. Numerical Implementation of the Exact Dynamics of Free Rigid Bodies, Journal of Computational Physics, 2007; 225(1): 145-164. https://doi.org/10.1016/j.jcp.2006.11.019

[10] Muller D. The Bizarre Behavior of Rotating Bodies, Explained. Veritasium. Retrieved February 2020; 16.

[11] Levi, Mark. Classical Mechanics with Calculus of Variations and Optimal Control: An Intuitive Introduction. American Mathematical Society. 2014; pp. 151-152. https://doi.org/10.1090/stml/069/07

[12] Usubamatov R. Theory of Gyroscopic effects for rotating objects, Springer, Singapore 2020. https://doi.org/10.1007/978-981-15-6475-8

[13] Usubamatov R. Inertial Forces Acting on Gyroscope, Journal of Mechanical Science and Technology, 2018; 32 (1): pp. 101-108. https://doi.org/10.1007/s12206-017-1211-0

[14] Taylor JR. (2005) Classical Mechanics. University Science Books, Mill Valley, CA. USA.

[15] Hibbeler RC. and Yap KB (2013) Mechanics for Engineers Statics and Dynamics, 13th ed. Prentice Hall, Pearson, Singapore.

[16] Gregory DR. (2006) Classical Mechanics. Cambridge University Press, New York.

[17] Syngley J and Uicker JJ. (2002), Theory of Machines and Mechanisms. 3ed., McGraw-Hill Book Company, New York.

[18] Aardema MD. (2005), Analytical Dynamics. Theory and Application. Academic/Plenum Publishers. New York. 